\title{
Expression Profile of Tumor Endothelial Marker 7 and a Putative Ligand in the Rat Spinal Cord and Dorsal Root Ganglion
}

\author{
Lih Wang ${ }^{*}$ Kyu-Yeol Lee*, Hwan-Tae Park ${ }^{\dagger}$, Dong-Sik Kang* \\ Departments of *Orthopedic Surgery, ${ }^{\dagger}$ Physiology, College of Medicine, Dong-A University, Busan, Korea
}

\begin{abstract}
Study Design: To analyze the expression profile of tumor endothelial marker 7 (TEM7) in the spinal cord and dorsal root ganglion (DRG).
\end{abstract}

Purpose: To investigate the expression profile of TEM7 in the spinal cord and DRG of adult and developing rats.

Overview of Literature: Tumor endothelial marker 7 (TEM7) is a putative transmembrane protein that is highly expressed in the tumor endothelium and in cerebellar neurons.

Methods: In the present study, the expression profile of TEM7 in the spinal cord and DRG of the rat was investigated using in situ hybridization and immunohistochemical analysis. In addition, the secreted recombinant ectodomain of TEM7 was employed to label the expression of a putative ligand of TEM7 in the spinal cord and DRG.

Results: Specific TEM7 mRNA localization was observed in the motor neurons of the spinal cord and sensory neurons of the DRG. Glial cells and vascular endothelial cells did not show hybridization signals. Immunohistochemical analysis with a specific polyclonal antibody revealed a similar localization profile for TEM7 mRNA expression. In the spinal cord, weak labeling was observed in the gray matter. The TEM7 ectodomain localized the expression of a putative ligand of TEM7 in the neurilemmal structures and perineurium of the spinal nerve roots. In the DRG, ligand labeling was observed in the endoneurium and perineurium of the spinal nerves, and extracellular matrix around the sensory neurons. A developmental study has shown that TEM7 mRNA expression in the motor neurons of the spinal cord and DRG increased with age during postnatal development.

Conclusion: These findings indicate that TEM7 plays a role as a transmembrane receptor in neuronal populations of the spinal cord and DRG.

Key Words: Tumor endothelial marker, Spinal cord, Dorsal root ganglion, Ligand

\section{Introduction}

Tumor endothelial markers (TEMs) are a group of cell surface proteins specifically expressed in the endothelium of various cancers, but are not expressed in normal endothelial tissues ${ }^{1-3}$. The limited expression profile of TEMs in the tumor endothelium indicates specific and restricted functions of the proteins in tumor angiogenesis ${ }^{2}$. TEMs associated with the cell surface are particularly of interest in that cell surface targets are directly accessible via the blood stream for functional intervention.

Several recent studies have showed evidence implicating a role of TEMs in normal tissues. For example, TEM1 is the lectin-like cell surface protein endosialin that is normally expressed in several peripheral tissues ${ }^{2}$, and TEM8 has been recently identified as the receptor for anthrax toxin ${ }^{4}$. It is also known that the mouse homologues of TEM1, TEM5 and TEM 8 are abundantly expressed in the endothelium of the embryonic liver ${ }^{2}$. Therefore, it appears that TEMs participate in a normal physiological process as well as in tumor angiogenesis.

Corresponding author: Kyu-Yeol Lee, MD

Department of Orthopedic Surgery, College of Medicine, Dong-A University

3-1 Dongdaesin-dong, Seo-gu, Busan, 602-715, Korea

Tel: +82-51-240-2867, Fax: +82-51-243-9764, E-mail: gylee@mail.donga.ac.kr 
Among the uncharacterized TEMs, TEM7 is a single transmembrane protein that contains the plexin-semaphorinintegrin (PSI) domain in its presumptive extracellular region $^{2}$. The PSI domain is mainly found in cell surface proteins of the nervous system, such as semaphorin and its receptor, plexin. The semaphorin family is a major repellent that regulates axon guidance and neuronal migration in the developing nervous system ${ }^{5,6}$. Interestingly, these neuronal guidance molecules having a PSI domain play an important role outside the nervous system, especially in the vascular system. For example, it was recently reported that semaphorin-3F and its receptor, neuropilin, regulate angiogenesis in vitro and in vivo ${ }^{7-10}$. Reversely, certain angiogenic factors appear to have an important function in the nervous system. For example, VEGF is known to regulate neuronal cell migration, neurite maturation and neurogenesis ${ }^{11-13}$. In terms of TEM7, Carson-Walter et al. ${ }^{2}$, recently demonstrated the presence of TEM7 mRNA in cerebellar Purkinje cells of the mouse brain, implicating a possible role of the endothelial protein, TEM7, in the nervous system. In the present study, to determine the expression profile and function of TEM7 in the spinal nerves, the histochemical localization of TEM7 in the spinal cord and dorsal root ganglion (DRG) of developing rats and adult rats was investigated.

The PSI domain, which was originally considered as a part of the sema domain, is found in several transmembrane proteins ${ }^{14}$. Even though the presumptive function of the PSI domain is protein binding ${ }^{14,15}$, biological evidence for a function of the PSI domain is still lacking. Most PSI proteins are type I transmembrane proteins that function as receptors or secreted ligands. The predicted structure of TEM7 suggests that it is also a type I transmembrane protein with a large extracellular region (ectodomain), a hydrophobic transmembrane domain, and a short cytoplasmic tail'2. A secreted form of the ectodomain of type I transmembrane proteins fused with a tag has been created to localize the expression of the corresponding ligands of the transmembrane proteins ${ }^{16}$. The present study employed the same strategy to analyze the expression profile of a putative ligand of TEM7 in the spinal cord and DRG using a recombinant TEM7 ectodomain.

\section{Materials and Methods}

\section{1. cRNA probes and in situ hybridization immunohis- tochemistry}

For producing a TEM7 cRNA probe, a cDNA fragment of rat TEM7 was used as a template. The plasmid containing the fragment of rat TEM7 cDNA was linearized at $\mathrm{NcoI}$ or Sall sites located in the polylinker for antisense and sense probes, respectively.

Labeling of RNA probes with digoxigenin-11-UTP (DIG11-UTP) was performed using an in vitro transcription kit (Roche Diagnostics). The amount and size of the transcribed cRNA were estimated with RNA gel electrophoresis.

In situ hybridization was performed as previously described with minor modifications ${ }^{17,18}$. Briefly, frozen spinal cords of adult Sprague-Dawley rats (p50 70, $\mathrm{n}=10$ ) were sectioned at $18 \mu \mathrm{m}$ thicknesses on a cryostat (Leica). For developmental analysis, Sprague-Dawley rat pups $(\mathrm{n}=12$ in total) were sacrificed at postnatal day 3,10 , and 20. The spinal cord and DRG were carefully dissected under a stereomicroscope (Olympus), and the central and peripheral branches of the DRG were removed with a fine scissor. The damaged DRG was discarded, and the intact DRG and spinal cord were frozen with dry ice. Frozen sections were prepared with the cryostat, and the sections were fixed in $4 \%$ paraformaldehyde (PFA) for 10 minutes, washed three times with phosphate buffered saline (PBS), and finally acetylated for 10 minutes. After prehybridization, the sections were incubated with hybridization buffer (50\% formamide, $4 \times$ SSC, $0.1 \%$ CHAPS, $5 \mathrm{mM} \mathrm{Na}_{2}$ EDTA, $0.1 \%$ Tween-20, $1.25 \times$ Denhartdt's, $125 \mu \mathrm{g} / \mathrm{mL}$ yeast tRNA, $50 \mu \mathrm{g} / \mathrm{mL}$ heparin, $200 \mathrm{ng}$ digoxigenin-labeled probes) for 18 hours at $57^{\circ} \mathrm{C}$. Non-specific hybridized proteins were removed by washing in $2 \times \mathrm{SSC}$ for 10 minutes and with RNase A (10 $\mu \mathrm{g} / \mathrm{mL})$, treatment), followed by a final washing step in $0.1 \times \mathrm{SSC}$ at $57^{\circ} \mathrm{C}$ for 15 minutes. For immunological detection of digoxigenin-labeled hybrids, the sections were incubated with anti-digoxigenin alkaline phosphatase (1:1500; Roche Diagnostics) for 1 hour, and the color reaction was carried out with 4-nitroblue tetrazolium chloride (NBT) and 5-bromo-4-chloro-3-indolyl phosphate (BCIP). Sections were dehydrated and were mounted with Crystalmount.

\section{Molecular cloning of the TEM7 ectodomain}

The ectodomain of human TEM7 (24aa-412aa) was generated using the polymerase chain reaction (PCR) with a human TEM7 cDNA template (a gift from Dr. B. Vogelstein). The sense PCR primers contained an SfiI site whereas the antisense primers had HindIII sites. After restriction enzyme digestion, the PCR fragments were inserted into the 
AP5 vector (a gift from Dr. Z. He) which contains alkaline phosphatase (AP), Myc and His as expression tags.

\section{Expression and purification of the TEM7 ectodomain}

HEK 293 cells were maintained in Dulbecco's modified Eagle's medium (DMEM) supplemented with $10 \%$ fetal bovine serum and antibiotics. The cells were transfected with $10 \mu \mathrm{g}$ of the expression vectors by the calcium phosphate method. The conditioned medium and transfected cells were harvested two days after transfection, and were solubilized with $2 \times$ sodium dodecyl sulfate (SDS) loading dye. The samples were subjected to SDS polyacrylamide gel electrophoresis and were transferred onto a nitrocellulose membrane (Hybond-ECL, Amersham). After blocking in 5\% non-fat milk in phosphate-buffered saline (PBS) containing $0.05 \%$ Tween 20 (PBST), the same membranes were incubated with anti-His or anti-Myc antibodies overnight at $4^{\circ} \mathrm{C}$. Next, the membranes were incubated with horseradish peroxidase conjugated anti-mouse or anti-rabbit IgG (1:3000; Amersham) for $1 \mathrm{~h}$ at room temperature. An enhanced chemiluminescence reaction (Amersham) was performed to visualize the reaction, and the blot was analyzed using a luminescent image analyzer (LAS-3000; Science Lab).

Individual AP fusion proteins were purified with nickelnitrilotriacetic acid beads. Conditioned medium from the transfected HEK 293 cells was passed over the nickel charged resins, and unbound proteins were eluted with buffer B $(500 \mathrm{mM} \mathrm{NaCl}, 20 \mathrm{mM}$ Tris- $\mathrm{HCl}, 5 \mathrm{mM}$ imidazole, $\mathrm{pH}$ 7.9) and buffer $\mathrm{W}(500 \mathrm{mM} \mathrm{NaCl}, 20 \mathrm{mM}$ Tris$\mathrm{HCl}, 60 \mathrm{mM}$ imidazole, $\mathrm{pH}$ 7.9). The AP fusion proteins were recovered by elution with buffer $\mathrm{E}(500 \mathrm{mM} \mathrm{NaCl}, 20$ $\mathrm{mM}$ Tris-HCl, $1 \mathrm{M}$ imidazole, $\mathrm{pH}$ 7.9), and were concentrated using Vivaspin concentrators (Viva science). SDSPAGE and Coomassie blue staining confirmed purity (> $90 \%)$.

\section{Measurement of alkaline phosphatase activity}

To measure of the activity of tagged AP of the fusion protein, conditioned media from stably transfected cells or from control HEK cells were used. The conditioned media were heated at $65^{\circ} \mathrm{C}$ for 10 minutes to inactivate the endogenous AP activity, and were then centrifuged at $13,000 \mathrm{rpm}$ for 5 minutes. The supernatant was incubated with $200 \mu \mathrm{L}$ of $1 \times$ AP buffer $(1.65 \mathrm{mg} / \mathrm{mL}$ p-nitro phenyl phosphate, $0.5 \mathrm{mM} \mathrm{MgCl}$, $0.1 \mathrm{M}$ diethanolamine (pH 9.8) for the indicated time, and the amount of color reaction was measured using a spectrophotometer at a wavelength of 405 nm.

\section{Immunohistochemistry}

Adult male Sprague-Dawley (p50 70, $n=20$ ) were used for immunohistochemical analysis and the frozen ligand binding assay. All procedures were performed according to protocols approved by the Dong-A University Committee on animal research that follow the guidelines of animal experimentation established by The Korean Academy of Medical Sciences. Animals were deeply anesthetized with an intraperitoneal injection of phentobarbital and were perfused with ice-cold PBS (pH 7.2), followed by 4\% PFA in PBS. The brains, spinal cord and dorsal root ganglia were removed and cryoprotected with $30 \%$ sucrose overnight and cryostat sections $(30 \mu \mathrm{m})$ were subsequently prepared. Tissue sections were permeated with $0.2 \%$ Triton X-100 in PBS for 15 minutes. After blocking with 10\% normal goat serum for 1 hour at room temperature, the sections were incubated with polyclonal anti-TEM7 antibody (1:2500) overnight at $4^{\circ} \mathrm{C}$. Sections were washed three times with PBS, and then sections were incubated with biotinylated anti-rabbit $\operatorname{IgG}$ (1:200; Vector Laboratories) for $2 \mathrm{~h}$ at room temperature. The sections were washed three times with PBS and were incubated with a mixture of avidin-biotin in PBS (1:100) for $1 \mathrm{~h}$ at room temperature. The chromogenic reaction was carried out with $0.05 \%$ diaminobenzidine and $0.03 \%$ hydrogen peroxide in $0.05 \mathrm{M}$ Tris- $\mathrm{Cl}$ buffer $(\mathrm{pH}$ 7.4). Sections were dehydrated through a series of graded alcohol, cleared with xylene, and mounted with Crystalmount.

\section{Ligand binding assay in tissue sections}

Frozen sections were fixed in 4\% PFA in PBS for 10 minutes at room temperature, washed twice in PBS for 5 minutes, and were then rinsed in ice-cold Hank's balanced salt solution (HBSS) containing $20 \mathrm{mM}$ hepes for $10 \mathrm{~min}$ utes. The sections were incubated with conditioned medium containing TEM7-AP or AP for 1 hour at room temperature, washed twice in HBSS for 10 minutes, and then fixed in AP fix solution $(65 \%$ acetone, $3 \%$ formaldehyde, $20 \mathrm{mM}$ Hepes, $\mathrm{pH}$ 7.5). Next, the sections were heated in PBS for 2 hours at $65^{\circ} \mathrm{C}$, rinsed in AP buffer $(100 \mathrm{mM}$ Tris- $\mathrm{HCl}, \mathrm{pH}$ 
9.5, $100 \mathrm{mM} \mathrm{NaCl}, 5 \mathrm{mM} \mathrm{MgCl}_{2}$ ) for 10 minutes to inactivate the endogenous alkaline phosphatase. Sections were developed with a developing solution containing NBT and BCIP for 5 10 minutes. Sections were then dehydrated through an ethanol series (70, 90, and $100 \%$ ethanol for 2 minutes each) and cleared twice in xylene. The sections were mounted with Crystalmount and were analyzed using a Zeiss Axiophot microscope.

\section{Results}

\section{Localization of TEM7 mRNA in the spinal cord and dorsal root ganglion}

The expression of TEM7 mRNA in the spinal cord and DRG was analyzed with in situ hybridization using a digoxigenin-labeled antisense riboprobe. In the spinal cord, the antisense probe specifically hybridized with TEM7 mRNA in the neuronal population of the gray matter (Fig. 1B and 1C), whereas the sense probe did not hybridize with TEM7 mRNA (Fig. 1A). The specific signals were mainly observed in the motor neurons of the ventral horn, but other neurons in the gray matter, including the dorsal horn, showed a very low level of TEM7 mRNA expression. The hybridization signals were observed only in the cytoplasm but not in the nuclei of the motor neurons (Fig. 1C). The expression of TEM7 mRNA in the motor neurons was observed with the same pattern throughout the whole length of the spinal cord. There were no specific hybridization signals in the white matter of the spinal cord, meninges and blood vessels.

In the DRG, TEM7 mRNA was strongly expressed in the cytoplasm of sensory neurons, but the nuclei of the sensory neurons were devoid of hybridization signals (Fig. 1E and 1F). The expression of TEM7 mRNA in the DRG was found in cells with various sizes, implicating nonselective TEM7 expression in various sensory neurons in the DRG. The hybridization signals in the satellite cells, Schwann cells and the spinal nerves were negative. The analysis of the sections hybridized with the sense probe showed no specific signals in the DRG (Fig. 1D).

\section{Cloning and characterization of the TEM7 ectodomain}

The predicted ectodomain of human TEM7 (eTEM7) (Fig. 2A) was forcefully expressed in HEK 293 cells. The expression of eTEM7, which is expected to secrete a $~ 110$ KDa protein composed of a TEM7 ectodomain, alkaline
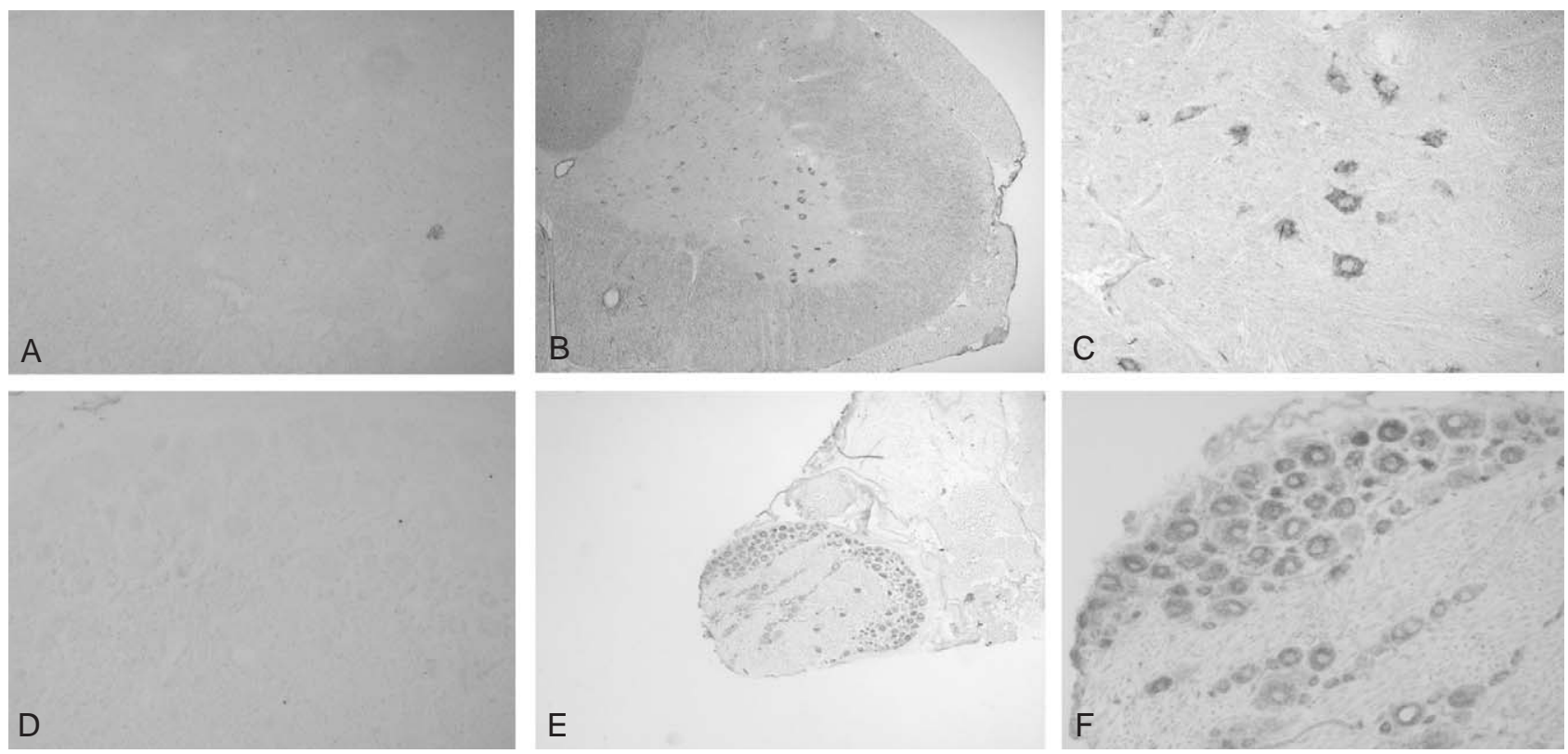

Fig. 1. Localization of TEM7 mRNA in the spinal cord and dorsal root ganglion. (A) Sense probe did not label any structures in the spinal cord. (B, C) TEM7 mRNA expression was observed in the neuronal cells in the gray matter of the spinal cord, especially in th motor neurons of ventral horn. (C) A representative high magnifcaton of the ventral horn of the spinal cord. (D) Sense probe did not label any structures in the dorsal root ganglion. (D, E) TEM7 mRNA expression was observed in the sensory neurons in the spinal cord. (F) A representative high magnification of the sensory neurons. 
phosphatase (AP; $67 \mathrm{KDa}$ ) (Fig. 2A) and Myc/His tags, occurred not only in cell pellets but also in the culture medium of transiently transfected cells (Fig. 2B). This indicates that TEM7 is a type I transmembrane protein that has a single extracellular domain with the N-terminal outside of the cell. The transfection of the empty vector (AP-5), which contains AP as a tag, also produced AP, and AP was observed both in the cell pellet and supernatant (Fig. 2B).

The activity of the tagged AP of TEM7 ectodomain was analyzed to demonstrate the utility of this fusion protein for localizing the expression of the TEM7 ligand. The controlled medium or purified TEM7 proteins were used to test AP activity. AP and TEM7-AP exhibited high AP activity as compared with the controlled HEK medium, and the activity was dose-dependent (Fig. 2C). This finding indicates that the secreted fusion protein is functionally active and that the construct could be a useful tool for analyzing the localization profile of the TEM7 ligand.

\section{Immunohistochemical analysis of TEM7 expres- sion in the spinal cord and dorsal root ganglion}

A polyclonal anti-TEM7 antibody that was purified with an affinity column was used to demonstrate the expression of TEM7 in the spinal cord and DRG at the protein level. The cerebellar section was used as a positive control for immunohistochemical analysis since TEM7 mRNA is known to be highly expressed in the cerebellar Purkinje cells $^{2}$. Consistent with the results of in situ hybridization, the antibody specifically labeled the cerebellar Purkinje cells, but not other cells such as granule cells (Fig. 3B). However, with the omission of the primary antibody, no cellular structures were labeled (Fig. 3A).

Next, immunohitochemical analysis of TEM7 protein expression in the spinal cord and DRG was investigated. The TEM7 antibody labeled neuronal cells especially in the ventral horn of the spinal cord (Fig. 3C and D). In the white matter of the spinal cord, the axoplasm seemed to show weak immunoreactivity. In the DRG, the anti-TEM7 antibody specifically labeled the sensory neurons and the immunoreactivity appeared in the cytoplasm, but not in the nuclei (Fig. 3E and F). The finding that the expression of TEM7 protein observed in almost all sensory neurons is consistent with the finding of TEM7 mRNA expression.

\section{Localization of a putative ligand of TEM7 in the spinal cord and dorsal root ganglion}

The expression of the putative ligand of TEM7 was analyzed by staining the binding site of the recombinant eTEM7-AP protein. In the spinal cord, the highest eTEMAP binding site was observed in the dorsal and ventral root
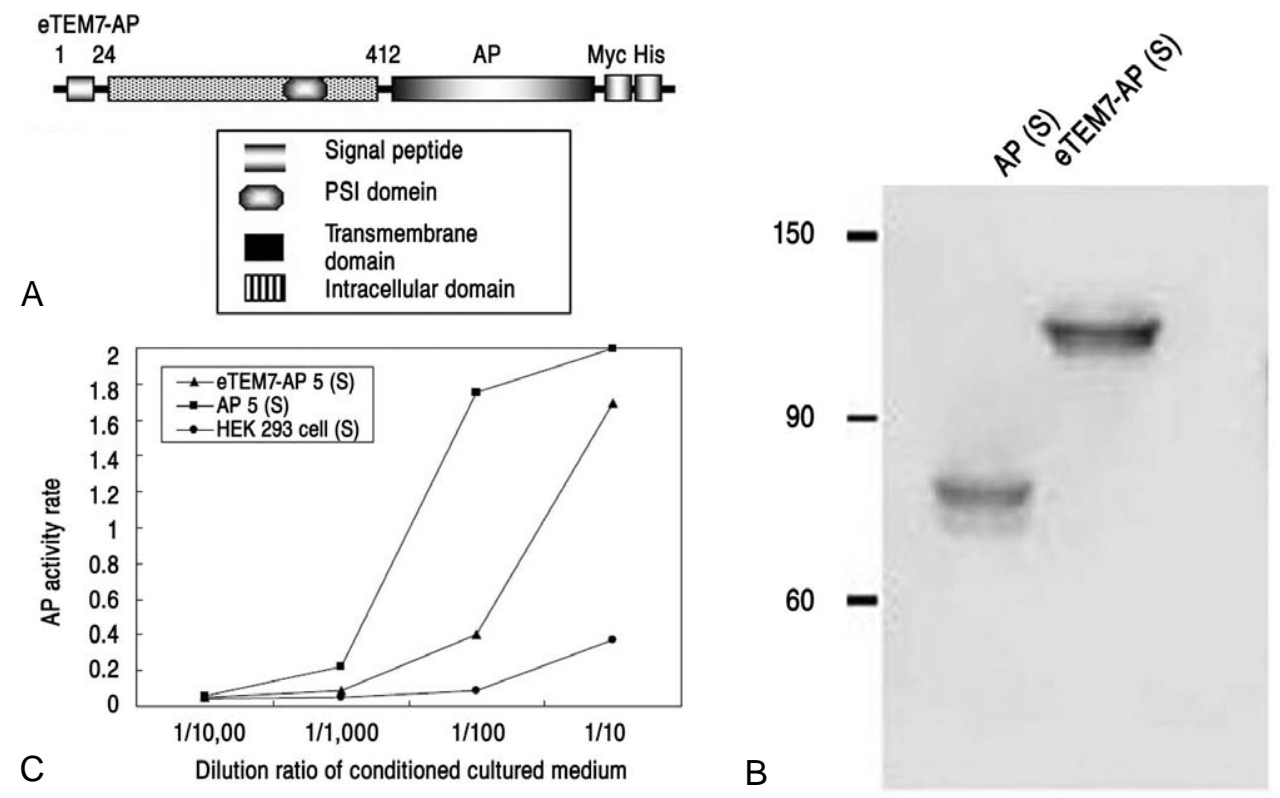

Fig. 2. Cloning and characterization of tumor endothelial marker 7 (TEM7) ectodomain. (A) Diagrammatic illustration of TEM7 constructs bases on amino acid sequence. AP: alkaline phosphatase. (B) The western blot showing the expression of TEM7 constructs in HEK cells. (C) AP activity assay showing a dose-dependent enzyme activity of tagged AP in the conditioned medium of transfected 293 cells. 

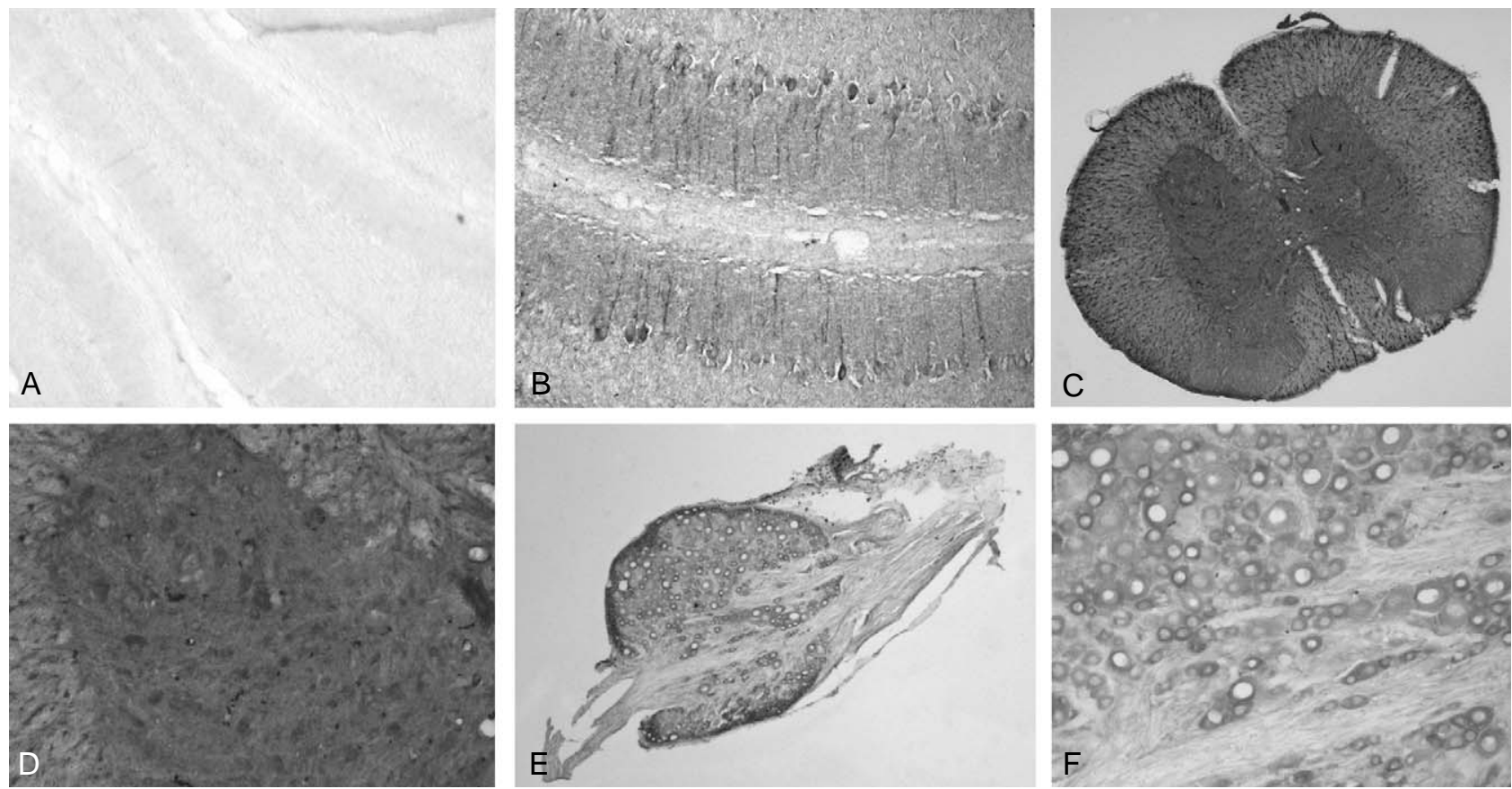

Fig. 3. Immunohistochemical analysis of the TEM7 expression. (A, B) Immunohistochemical stainings with an anti-TEM antibody in a cerebellar section show the expression of TEM7 in tjje cerebellar Purkinje cells. (B) The omission of the primary antibody did ont label any structures in the cerebellum. (C $\sim$ F) The anti-TEM7 antibody specifically labeled the neuronal cell bodies in the ventral horn o the spinal cord (C, D) and DRG $(\mathbf{E}, \mathbf{F})$. (F) The cytoplasm showed intense staining whereas nuclei were devoid of the staining in sensory neurons.
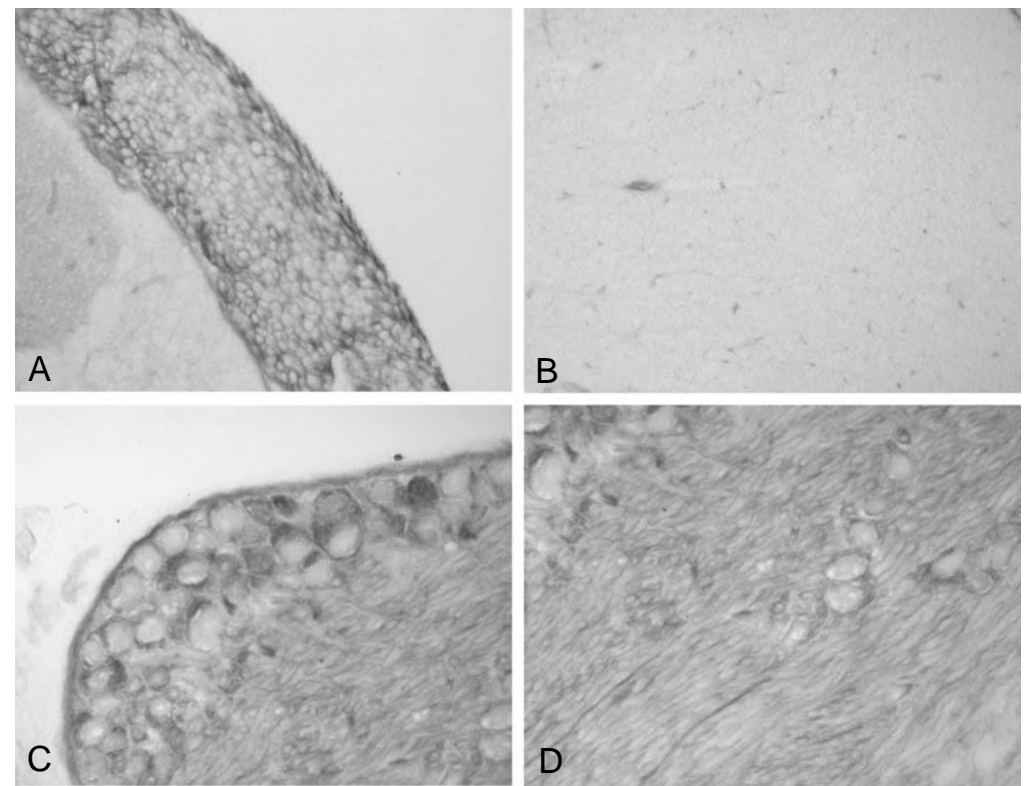

Fig. 4. Localization of a putaive ligand of TEM7 in the spinal cord and dorsal root ganglion. (A) A representative staining of eTEM$\mathrm{AP}$ in the dorsal root of the spinal nerves. Intense staining was observed in the perineural structures of the dorsal root, but low level of ligand binding was found in the gray matter. (B) In the while matter of the spinal cord, the ligand binding was observed in the blood vessels, not neural structures. (C) The strong binding reaction was found in the membrane of sensory neurons and spinal nerves in the DRG. (D) Perineural structures exhibited an obvious ligand binding.

of the spinal nerves (Fig. 4A). The binding seemed to be observed in the neurilemma and endoneurium, but not in the axoplasm, axolemma and myelin sheath. The perineurium that surrounds the nerve fascicle also showed the strongest eTEM7-AP binding. In the gray matter, a very low level of eTEM7-AP binding was observed (Fig. 4A), but the cell 

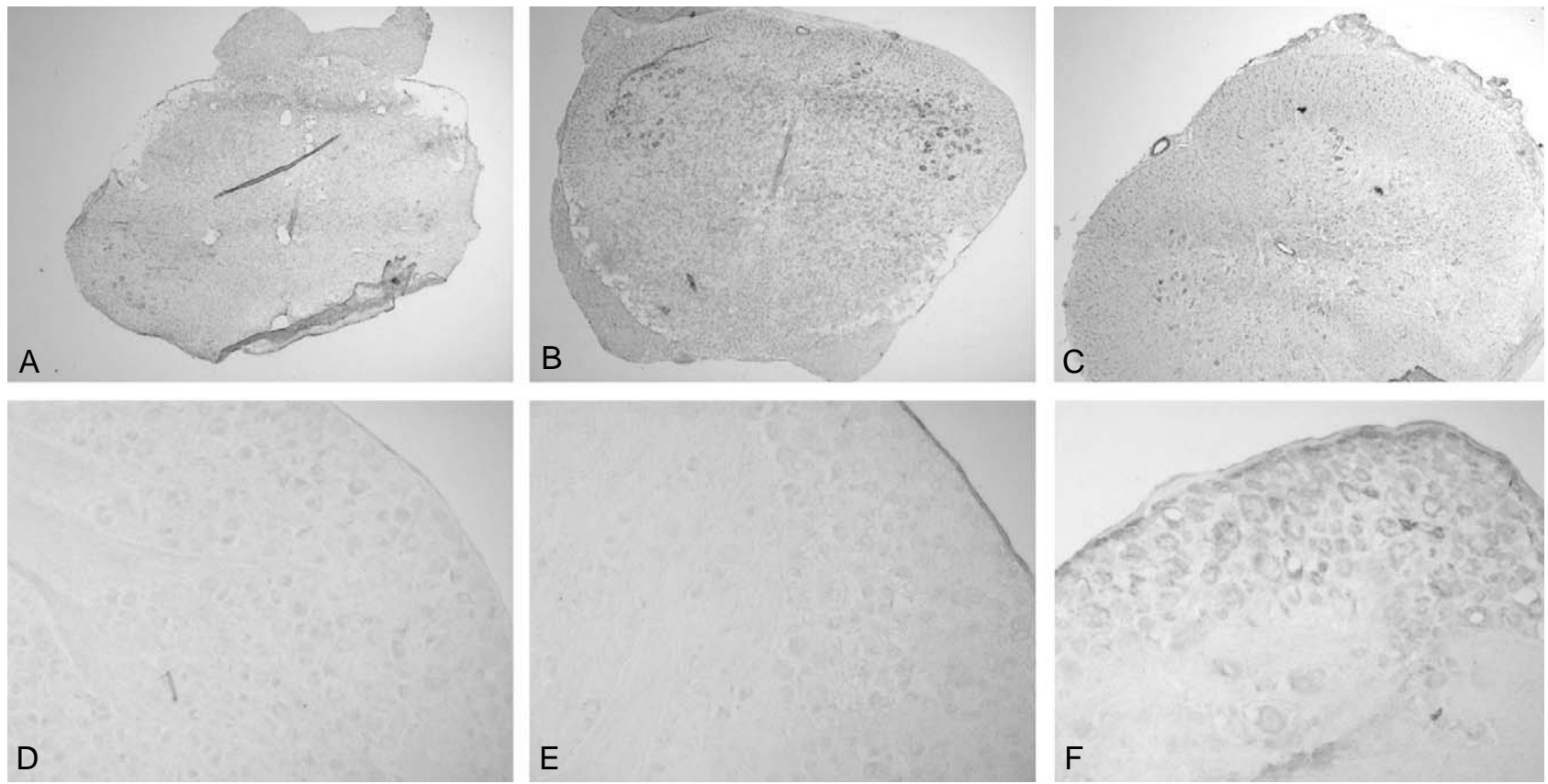

Fig. 5. Developmental expression of TEM7 mRNA in the spinal cord and dorsal root ganglion. (A C) TEM7 mRNA expression in the spinal cord during postnatal development. The expression of TEM7 mRNA was increased with ages from postnatal day 3 (A). An obvious TEM7 mRNA expression was observed in the neurons of the gray matter at postnatal day 10 (B) and 20 (C). (D F) TEM7 mRNA expression in the DRG during postnatal development. The expression of TEM7 mRNA was not observed in the DRG at postnatal day $3(\mathbf{D})$ and $10(\mathbf{E})$, but the mRNA expression was found at postnatal day $20(\mathbf{F})$.

bodies of the motor neurons which expressed TEM7 mRNA did not show any binding of eTEM7-AP. There was no labeling of eTEM7-AP in the neural structures of the white matter such as the axon fibers, myelin sheath, and extracellular matrix (Fig. 4B). However, specific binding of eTEM7-AP is clearly found in the wall of blood vessels (Fig. 4B). As a negative control, AP protein was used to stain the sections of the spinal cord. AP did not bind any structures in the spinal cord (data not shown).

In accordance with the strong reactivity of the neurilemmal structure of the spinal nerve roots to eTEM7-AP, the spinal nerves within the DRG also showed strong eTEM7AP binding (Fig. 4C and D). In addition, the plasma membrane of sensory neurons in the DRG had prominent eTEM7-AP binding activity (Fig. 4C).

\section{Developmental expression of TEM7 mRNA and its putative ligand in the spinal cord and dorsal root ganglion}

Using in situ hybridization, the developmental changes of TEM7 mRNA expression in the spinal cord and DRG were analyzed. TEM7 mRNA in the motor neurons of the spinal cord first appeared on postnatal day 3, but the expression level was very low (Fig. 5A). A significant increase of TEM7 mRNA was observed during second postnatal weeks (Fig. 5B and C). However, the glial cells and endothelial cells did not show expression of TEM7 mRNA throughout the postnatal developmental period. In the DRG, expression of TEM7 mRNA was not observed at postnatal day 3 and 10 (Fig. 5D and E). TEM7 mRNA expression in the sensory neurons in the DRG was obviously found at postnatal day 20, and expression was confined to the sensory neurons, and not in other structures (Fig. 5F).

The ligand binding assay showed that the TEM7 binding activity of the spinal nerves appeared as early as postnatal day 3 (Fig. 6A). The binding was mainly observed in the neurilemma of the nerve roots with a similar binding pattern of eTEM7-AP in the adult spinal cord. The intensity of the staining in the spinal nerve root did not significantly change with age (Fig. 6B). The gray matter showed a low level of ligand binding, but the white matter did not, during the postnatal period (Fig. 6A and B).

\section{Discussion}

The present study is the first to show neuronal expression 

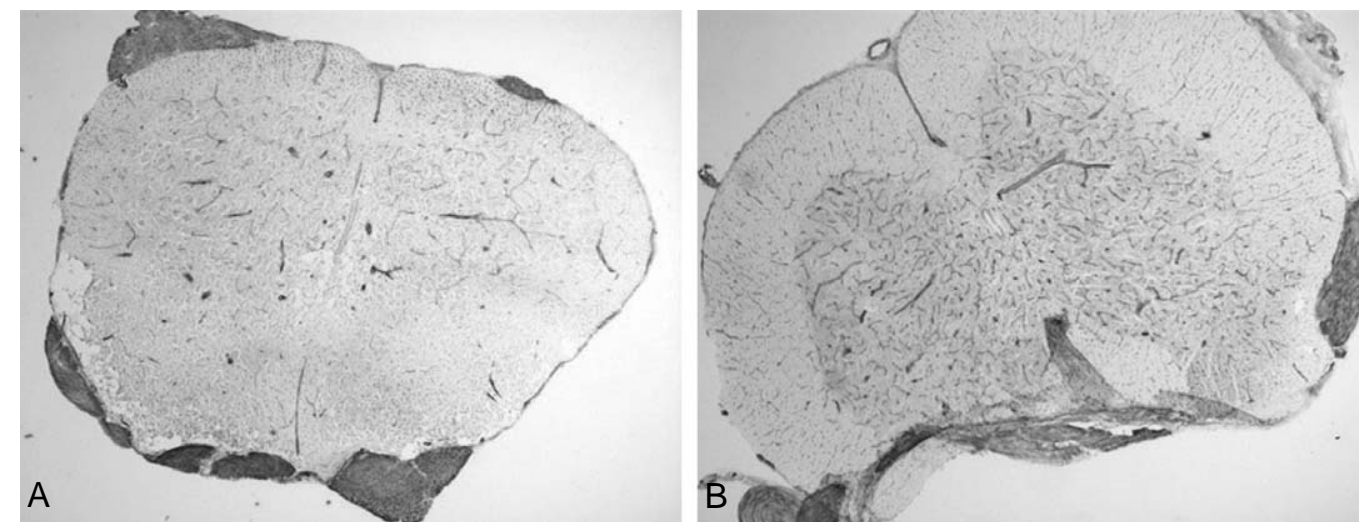

Fig. 6. Developmental expression of aeputative ligand for TEM7 in the spinal cord and spinal nerves. (A, B) A strong ligand binding was appeared at postnatal day 3 (A) and 20 (B) in the nerve roots of the spinal nerves. The gray matter of the spinal cord and blood vessel showed ligand binding sites at early postnatal days.

of TEM7 mRNA and protein in the spinal cord and DRG of rats. TEM7 mRNA expression was not observed in ependymal cells and endothelial cells of the spinal cord and DRG. mRNA expression in spinal neurons was demonstrated by immunohistochemical staining of TEM7 protein in motor and sensory neurons in the spinal cord and DRG, respectively. These findings indicate that TEM7 may play a specific role in several neuronal cells of the spinal nervous system.

The predicted structure of TEM7 is that of a type I transmembrane protein with a large extracellular region (ectodomain), a hydrophobic transmembrane domain, and a short cytoplasmic tail'2. A secreted form of the ectodomain of type I transmembrane proteins that was fused with a tag has been made to localize the expression of the corresponding ligands of the ectodomains ${ }^{16}$. The present study employed the same strategy to make a secreted form of TEM7 ectodomain, and the localization of the putative ligand in the spinal cord and DRG was investigated using a recombinant TEM7 ectodomain. We found that the coverings of spinal nerves, such as the neurilemma, endoneurium and perineurium, show strong ligand binding to the TEM7 ectodomain. In the DRG, the ligand binding appeared around the sensory neurons and perineural structures of the spinal nerves. This finding indicates that the putative ligand of TEM7 may be a perineurial extracellular matrix (ECM) protein or a secreted extracellular protein.

The PSI domain consists of $\sim 50$ residues of amino acids and usually contains eight cysteine residues ${ }^{14}$. The cysteine residues are expected to be disulfide bonded. Even though the presumptive function of the PSI domain is protein-binding $^{4,15}$, the biological function of the PSI domain is still unknown. Most PSI proteins are highly expressed during the developmental period in the nervous system, suggesting a role of the PSI domain-containing protein in the developmental processes such as axon guidance and neuronal migration. These developmental phenomena are tightly regulated by the interaction between the cell membrane receptor and ECM proteins. Therefore, the molecular structure of TEM7 and the localization profile of the putative TEM7 ligand suggest a possible role of TEM7 in neural development, especially in the spinal cord and DRG.

The majority of ECM proteins are structural proteins such as laminin and fibronectin ${ }^{19}$, and ECM proteins play a critical role in the development of various tissues. In the nervous system, ECM proteins regulate the adhesion and migration of growth cones and neuronal cells during development $^{20}$. The present study indicates that the TEM7 ligand may be a perineurial ECM molecule. In contrast to the strong staining of TEM7 ligand in the perineural structure of peripheral spinal nerves, there was no staining in the white matter of the spinal cord. This finding indicates that the putative TEM7 ligand is expressed in certain cells of the peripheral nervous system. It may be possible that Schwann cells that surround the peripheral nerves will produce the putative TEM7 ligand. As the basal lamina of Schwann cells provide important substrates not only for developing axons but also for regenerating axons ${ }^{21}$, the putative TEM7/ECM ligand interaction may play a role in neurite growth during development and/or regeneration.

The present study has also revealed that the expression of TEM7 mRNA in the spinal cord and DRG was developmentally regulated. The expression increased during postnatal development. In addition, the ligand binding assay showed expression of the putative ligand of TEM7 as early 
as postnatal day 3 in the spinal nerve fibers. This finding indicates that TEM7-ligand interaction plays a role in the postnatal differentiation of spinal motor neurons and sensory neurons of DRG. TEM7 mRNA expression in the Purkinje cells of the cerebellum also increased during postnatal development (preliminary observation). Therefore, it seems that TEM7 might play an important role in the postnatal development of vertebrate neurons. Further studies on the function of TEM7 and its ligand in spinal nerve development would provide an insight into a novel mechanism of spinal cord development.

Even though the present and previous studies have revealed the expression profile of TEM7 in the nervous system and tumor endothelial cells, the function of TEM7 in neurons and endothelial cells is completely unknown at present. TEM7 seems to be involved in tumor angiogenesis in concert with other TEMs. It has been recently reported that TEM8 binds to an ECM protein, collagen alpha $3^{22}$. The present study has revealed a possibility of an ECM protein as a TEM7 ligand. It was also reported that the TEM7 ectodomain inhibits fibrin-induced angiogenesis in vitro ${ }^{23}$, indicating a possible interaction between the TEM7 ectodomain and ECM matrix may regulate angiogenesis. Thus, the identification of TEM7 ligand will shed new light on the elucidation of TEM7 function in the nervous system as well as in tumor angiogenesis.

\section{Conclusions}

These findings indicate that TEM7 plays a role as a transmembrane receptor in neuronal populations of the spinal cord and DRG.

\section{REFERENCES}

1. St Croix B, Rago C, Velculescu V, et al: Genes expressed in human tumor endothelium. Science 2000; 289: 1197 1202.

2. Carson-Walter EB, Watkins DN, Nanda A, Vogelstein B, Kinzler KW, St Croix B: Cell surface tumor endothelial markers are conserved in mice and humans. Cancer Res 2001; 61: 6649-6655.

3. Nanda A, St Croix B: Tumor endothelial markers: new targets for cancer therapy. Curr Opin Oncol 2004; 16: 4449.
4. Bradley KA, Mogridge J, Mourez M, Collier RJ, Young JA: Identification of the cellular receptor for anthrax toxin. Nature 2001; 414: 225-229.

5. Raper JA: Semaphorins and their receptors in vertebrates and invertebrates. Curr Opin Neurobiol 2000; 10: 88-94.

6. Park HT, Wu J, Rao Y: Molecular control of neuronal migration. Bioessays 2002; 24: 821-827.

7. Kessler O, Shraga-Heled N, Lange T, et al: Semaphorin$3 \mathrm{~F}$ is an inhibitor of tumor angiogenesis. Cancer Res 2004; 64: 1008-1015.

8. Soker S, Takashima S, Miao HQ, Neufeld G, Klagsbrun $\mathbf{M}$ : Neuropilin-1 is expressed by endothelial and tumor cells as an isoform-specific receptor for vascular endothelial growth factor. Cell 1998; 92: 735-745.

9. Neufeld G, Cohen T, Shraga N, Lange T, Kessler O, Herzog Y: The neuropilins: multifunctional semaphorin and VEGF receptors that modulate axon guidance and angiogenesis. Trends Cardiovasc Med 2002; 12: 13-19.

10. Gu C, Rodriguez ER, Reimert DV, et al: Neuropilin-1 conveys semaphorin and VEGF signaling during neural and cardiovascular development. Dev Cell 2003; 5: 45-57.

11. Fabel K, Fabel K, Tam B, et al: VEGF is necessary for exercise-induced adult hippocampal neurogenesis. Eur $\mathbf{J}$ Neurosci 2003; 18: 2803-2812.

12. Zhang H, Vutskits L, Pepper MS, Kiss JZ: VEGF is a chemoattractant for FGF-2-stimulated neural progenitors. J Cell Biol 2003; 163: 1375-1384.

13. Khaibullina AA, Rosenstein JM, Krum JM: Vascular endothelial growth factor promotes neurite maturation in primary CNS neuronal cultures. Brain Res Dev Brain Res 2004; 148: 59-68.

14. Bork P, Doerks T, Springer TA, Snel B: Domains in plexins: links to integrins and transcription factors. Trends Biochem Sci 1999; 24: 261-263.

15. Zang Q, Springer TA: Amino acid residues in the PSI domain and cysteine-rich repeats of the integrin beta2 subunit that restrain activation of the integrin alpha(X)beta(2). J Biol Chem 2001; 276: 6922-6929.

16. Wang KC, Koprivica V, Kim JA, et al: Oligodendrocytemyelin glycoprotein is a Nogo receptor ligand that inhibits neurite outgrowth. Nature 2002; 417: 941-944.

17. Schaeren-Wiemers N, Gerfin-Moser A: A single protocol to detect transcripts of various types and expression levels in neural tissue and cultured cells: in situhybridization using digoxigenin-labelled cRNA probes. Histochemistry 1993; 100: 431-440.

18. Park HT, Kang EK, Bae KW: Light regulates Homer 
mRNA expression in the rat suprachiasmatic nucleus. Brain Res Mol Brain Res 1997; 52: 318-322.

19. De Arcangelis A, Georges-Labouesse E: Integrin and ECM functions: roles in vertebrate development. Trends Genet 2000; 9: 389-395.

20. Sobeih MM, Corfas G: Extracellular factors that regulate neuronal migration in the central nervous system. Int J Dev Neurosci 2002; 20: 349-357.

21. Chernousov MA, Carey DJ: Schwann cell extracellular matrix molecules and their receptors. Histol Histopathol 2000; 15: 593-601.

22. Nanda A, Carson-Walter EB, Seaman S, et al: TEM8 interacts with the cleaved $\mathrm{C} 5$ domain of collagen alpha 3(VI). Cancer Res 2004; 64: 817-820.

23. Wang XQ, Sheibani N, Watson JC: Modulation of tumor endothelial cell marker 7 expression during endothelial cell capillary morphogenesis. Microvasc Res 2005; 70: 189197. 